\title{
A Review Paper on Image Inpainting and their Different Techniques
}

\author{
Pranjali Joshi $^{1}$ and Neeraj Shrivastav ${ }^{2}$ \\ ${ }^{1}$ PG Student, ${ }^{2}$ Associate Professor, \\ Computer Science and Engineering, Institute of Engineering and Science, \\ Institute of Professional Studies, Indore, Madhya Pradesh, India \\ E-Mail: pranjalijoshi1992@gmail.com
}

\begin{abstract}
Image Inpainting is an art of modifying the digital image in such a way that the modifications are undetectable to an observer who has no idea about the original image. The essential thought behind the system is to consequently fill in lost or missing parts of a picture utilizing data from the encompassing region. It is utilized for rebuilding of old movies and protest expulsion in computerized photos. Different calculations have introduced in the past to accomplish the undertaking of picture inpainting. In this paper we give a survey of various systems utilized for picture Inpainting. We talk about various inpainting systems like Wavelet Transform inpainting, Exemplar based picture inpainting, PDE based picture inpainting, surface combination based picture inpainting.

Keywords: Image inpainting, Image processing, Exemplar Based Inpainting, object removal, Noise, Image Reconstruction
\end{abstract}

\section{INTRODUCTION}

In recent year, digital image inpainting is one of the interesting new research topics in the field of image processing, which can be used in various applications like computer graphics, image editing, film post production, image restoration etc. [1].

Image inpainting is an ancient art. There are heaps of focal points sight and sound instruments in this day and age people groups are clicking loads of Picture or pictures of theirs and furthermore endeavoring to save their past pictures. What's more, as the time goes on those photos got harmed (breaks, starches, picture information misfortune, undesirable and so forth.) Inpainting is the craft of reestablishing lost parts of a picture and recreating them in light of the foundation data. In genuine world, numerous individuals require a framework to recoup harmed photos, outlines, illustrations, craftsmanships and so forth harm might be because of different reasons like scratches, overlaid content or designs and so on. Inpainting system has numerous applications, for example, protest evacuation in advanced photographs, expulsion of impediments (date ,stamps , logo and so on, for example, huge undesirable districts, red eye revision, super determination, rebuilding of old movies and compositions etc.[2] [3].

Picture inpainting is a developing zone of picture handling; with the assistance of this we can fill the lost or lost locales of a given picture. All things considered, for instance in the exhibition hall world the activity of inpainting of valuable pictures or artworks is satisfied with the assistance of a specialist of expressions. In light of the establishment information, picture inpainting try to fill the degraded/missing data in the photo. Picture reinstallation includes re-establishment of old pictures/photographs and hurt picture/film by end of clean spots, specks, scratches, superimposed substance like dates, subtitles, or notoriety. End of unfortunate things from a photograph/picture, for instance, transfer of a bothersome thing like a man, animal, tree et cetera is known as question clearing. The target of picture inpainting isn't simply to recover the photo, yet to make few pictures that have immovably equivalent with the principal picture [4].

This paper is an overview of image inpainting and their techniques and describes some of the commonly used techniques to solve complex problems with image inpainting methods.

Rest of the paper is organized as follow: in section II background study of image inpainting and their relevancy is done, in section III previous researches done in Image inpainting is described, section IV explain problem statement of the image inpainting. Lastly in section VII summarize the brief summary of the paper i.e. conclusion.

\section{BACKGROUND}

The foundation of an examination is a vital piece of our review paper. It gives the unique circumstance and reason for the examination. Henceforth there is requirement for foundation considers that adds to plan distinctive parts of the picture inpainting for picture based applications.

\section{A. Image Inpainting}

Picture inpainting is a craft of missing quality or an information in a picture. The motivation behind picture inpainting is to reproduce missing districts which is obvious for human eyes. Picture inpainting is the way toward remaking lost piece of pictures in light of the foundation data.

The change of pictures in a way that is non-perceivable for a spectator who does not know the first picture is training as old as aesthetic creation itself. The need to modify the picture in a subtle way stretched out normally from compositions to photography and film. The reasons continue as before to return disintegration (e.g., cracksin photos or 
scratches and tidy spots in film), or to include or evacuate components (e.g., expulsion of stamped date and red-eye from photos, the notorious "digitally embellishing" of political foes). Inpainting is the craft of reestablishing lost parts of a picture and reproducing them in view of the foundation data. This must be done in an imperceptible way. The term inpainting is gotten from the antiquated craft of reestablishing picture by proficient picture restorers in historical centers and so on. Advanced Image Inpainting endeavors to impersonate this procedure and play out the inpainting naturally [5].

Figure 1 demonstrate a case of this procedure where a building (physically chose as the objective district) is supplanted by data from the staying of the picture in an outwardly conceivable manner. The calculation consequently does this in a way that it looks "sensible" to the human eye. Subtle elements that are covered up/blocked totally by the question be expelled can't be recouped by any numerical strategy. Thusly the goal for picture inpainting isn't to recuperate the first picture, however to make some picture that has a nearby similarity with the first picture.

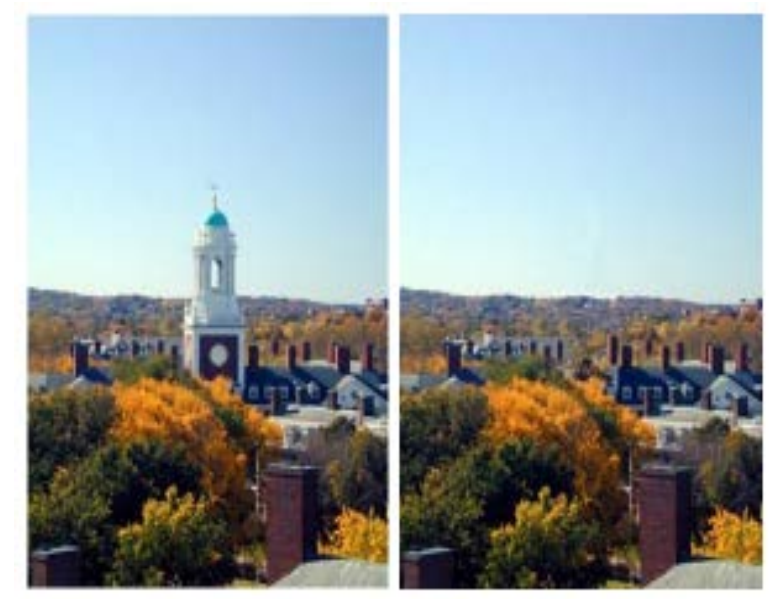

Fig. 1 Removing objects using Image Inpainting

In this figure, (a) The original image, (b) Picture with the building expelled. Notice how the form of mountain and the surfaces have both been revised.

\section{B. Image Inpainting Techniques}

Picture inpainting calculations can be grouped into various classes like surface union based picture inpainting, Exemplar and inquiry based picture inpainting, PDE (Partial Differential Equation) based inpainting, Fast self-loader inpainting and half and half inpainting. Here in this area we have clarified these inpainting strategies [6] [7] [8].

1. Partial Differential Equation (PDE) based: The calculation is to proceed geometric and photometric data that touches base at the fringe of the blocked zone into region itself. This is finished by spreading the data toward insignificant change utilizing is ophotelines. This calculation will create great outcomes if missed locales are little one.
2. Texture combination based: The Texture union is a field of concentrate free from, however identified with inpainting. In the general meaning of this issue, an information test of a surface is given, and the objective is to create a greater amount of that surface. The least complex arrangement is to tile the surface example on a rectangular lattice of wanted size.

3. Exemplar and pursuit based: The model based comprises of two essential advances, 1.priority task is done and, 2. the choice of the best coordinating patch. The model based approach tests the best coordinating patches from the known district, whose similitude is estimated by specific measurements, and glues into the objective fixes in the missing area. Model based Inpainting iteratively combines the obscure district, I. e. target area, by the most comparable fix in the source district. All in all, model based inpainting calculation is made out of following advances.

a. Initial blocked area is recognized and are spoken to with the information structures that are fitting to the locale.

b. A predefined work is utilized to process the taking care of request for all the missing pixels as the initial phase in every one of the cycles.

c. From the source district, the most coordinating square is sought to settle the given impeded locales hinder with a pixel trotted at p.

d. Boundary of the objective district and the data for processing filling needs are refreshed.

4. Wavelet Transform based: We expect the best worldwide structure estimation of harmed districts fit as a fiddle and surface properties. On the off chance that we consider the reality of multi-determination investigation, information partition, compaction alongside the factual properties then we need to consider the wavelet change because of its great picture portrayal quality. Wavelet change endeavor to fulfill the human visual framework (HVS)

5. Semi-automatic and Fast Inpainting: This picture in painting needs client support the as rules to help in structure culmination has discovered support with specialists. This strategy brings about obscure impact in picture.

\section{Modeling and Computation of Lower Level Inpainting Algorithms}

The general rules which can be exceptionally useful in making novel commitments in demonstrating and calculation of lower level inpainting calculations are [9] [10]:

1. Locality: Inpainting is neighborhood. The missing data is inpainted construct just with respect to picture data accessible in the regions of missing zones.

2. Functionality: Inpainting is useful. The inpainting models or calculations depend just on properties of pictures as capacities and not on any larger amount design acknowledgment inputs. 
3. Automation: Inpainting must be as programmed as would be prudent. The lesser human info requests, all the more intense models for genuine application

4. Generality: Inpainting ought to have the capacity to manage whatever number bland pictures as could be expected under the circumstances. It implies that as long as data lost is neighborhood, the most non specific deficient pictures can be effectively inpainted to certain palatable accuracy.

5. Stability: Inpainting ought to be steady, implying that it more likely than not inherent instruments to oppose any minor debasement of the accessible picture data, for example, clamor and obscure, which are exceptionally regular in applications

\section{LITERATURE SURVEY}

This section provides the recently made contribution and the research work performed for improving image quality by mean of implemented image inpainting. Thus different research articles and papers are included in this section.

Bin Dong et al., [11] introduced a visually impaired inpainting model to tackle this kind of issues, i.e., a model of at the same time distinguishing and recouping harmed pixels of the given picture. A tight casing based regularization approach is created in this paper for such visually impaired inpainting issues, and the came about minimization issue is unraveled by the part Bregman calculation. The proposed dazzle inpainting technique is connected to different testing picture reclamation undertakings, including recuperating pictures that are foggy and harmed by scratches and expelling picture commotion blended with both Gaussian and arbitrary esteemed drive clamor. The examinations demonstrate that this technique is looked at positively against numerous accessible twoarranged strategies in these applications.

Junyuan Xie et al., [12] displayed a novel way to deal with low-level vision issues that joins scanty coding and profound systems pre-prepared with denoising auto-encoder (DA). Creators proposed an elective preparing plan that effectively adjusts DA, initially intended for unsupervised component learning, to the undertakings of picture denoising and dazzle inpainting. This current strategy's execution in the picture denoising errand is practically identical to that of KSVD which is a broadly utilized scanty coding procedure. All the more critically, in dazzle picture inpainting undertaking, the proposed strategy gives answers for some unpredictable issues that have not been handled previously. In particular, creators can naturally expel complex examples like superimposed content from a picture, as opposed to basic examples like pixels missing aimlessly. In addition, the proposed technique does not require the data with respect to the locale that requires inpainting to be given from the earlier. Trial comes about exhibit the adequacy of the proposed technique in the errands of picture denoising and dazzle inpainting. Creators likewise demonstrate that our new preparing plan for DA is more viable and can enhance the execution of unsupervised element learning.

Weisheng Dong et al., [13] adopt a low-rank strategy toward SSC and give a thoughtfully basic understanding from a reciprocal difference estimation viewpoint, to be specific that specific regard rot of similar squeezed patches can be seen as pooling both neighborhood and nonlocal data for evaluating signal fluctuations. Such viewpoint motivates us to build up another class of picture reclamation calculations called spatially versatile iterative solitary esteem thresholding (SAIST). For clamor information, SAIST sums up the observed BayesShrink from neighborhood to nonlocal models; for deficient information, SAIST expands past deterministic strengthening based answer for sparsity streamlining through joining the possibility of word reference learning. Notwithstanding applied straightforwardness and computational productivity, SAIST has accomplished exceedingly skillful (regularly better) target execution contrasted with a few cutting edge strategies in picture denoising and finish tests. Consequently, subjective quality outcomes contrast positively and those acquired by existing systems, particularly at high commotion levels and with a lot of missing information.

Qing Zhang et al., [14] proposed a novel model construct inpianting calculation in view of the shading dispersion examination. A novel need plot is intended to allot need and guide the dispatching all together, which is an essential advance for the execution of the inpainted comes about. Shading dissemination is proposed to decide the certainty of the objective patches arranged on the limit of the missing district by estimating the variety of differences of neighboring source patches. This trials on the regular pictures and the examinations with the agent existing methodologies demonstrate that our proposed plan would more be able to powerfully allocate high need to the structures and empowers the notable structures, to which human visual framework is touchy, to be reliable with the encompassing locales.

Anamandra Sai Hareesh et al., [15] propose a quick and straightforward procedure in view of a novel inclination capacity and its speculation through partial subordinates to assess the taking care of request prioritization. Results show better and vigorous execution over all the ongoing advances in the space of model based strategies cited in the writing.

Yunqiang Liu et al., [16] presented a novel definition of model based inpainting as a worldwide vitality improvement issue, written as far as the counterbalance outline. The proposed vitality useful consolidates an information connection term to guarantee the congruity of the recreation at the limit of the inpainting area and a smoothness term that guarantees an outwardly intelligent reproduction inside the gap. This plan is adjusted to acquire a worldwide least utilizing diagram cuts calculation. Keeping in mind the end goal to lessen the computational multifaceted nature, we propose an effective multiscale 
chart cuts calculation. In addition, to make up for the loss of data at low determination levels we utilize an element portrayal processed at the first picture determination. These licenses to reduce the uncertainty initiated by contrasting just shading data when the picture is spoken to at low determination levels. Our investigations demonstrate the great execution of the proposed calculation when contrasted and other ongoing calculations.

\section{PROBLEM DOMAIN}

The most common problem of the Exemplar-based approaches is that, in some cases, acceptable results cannot be obtained because large missing region is filled up by using small number pixels which are known to us. The patch shifting approach makes sure that the patch that has to be fill up will always contains enough number of known pixels so that it can productively contrast and the hopeful fix in the source locale. Along these lines, most solid fix can be filled the obscure area. In powerful inpainting approach utilizing locale division, division delineates graphical representation of local texture and represent missing region with white. With the information available about the segmented image map, the proposed approach decides the reasonable fix estimate and chooses the best match fix from the source locales for diminishing unnatural ancient rarity.

\section{CONCLUSION}

This paper deal with different terminology of the image inpainting paradigm for image processing based application. Additionally, in this paper we described various image inpainting techniques which are solely used in real time environment. From this examination, various deficiencies and constraints were featured of these systems. It is watched that the PDE based Inpainting calculations can't fill the expansive missing area and it can't modify the surface example. The hypothetical examination demonstrated that model based Inpainting will create great outcomes for Inpainting the substantial missing district additionally these calculations can inpaint both structure and finished picture also. In addition, our examination involves development of productive calculation to diminish computational cost and to diminish the time required for Inpainting.

\section{REFERENCES}

[1] Jaspreet Kaur Chhabra and Vijay Birchha, "An Enhanced Technique for Exemplar based Image Inpainting", International Journal of Computer Applications (IJCA), Vol. 115, No. 17, April 2015.

[2] Ankur G. Patel, Shashwat kumar and Ankit D. Prajapati, "Analysis of Exemplar Based Image Inpainting”, IJCSIT) International Journal of Computer Science and Information Technologies, Vol. 5, No. 1, pp. 800-804, 2014.

[3] M Bertalmio, L Vese, G Sapiro and S.Osher, "Simultaneous structure and texture image inpainting," IEEE Transactions on Image Processing, Vol. 12, pp. 882-889, 2003.

[4] Jaspreet Kaur Chhabra and Mr. Vijay Birchha, "Detailed Survey on Exemplar Based Image Inpainting Techniques”, (IJCSIT) International Journal of Computer Science and Information Technologies, Vol. 5, No. 5, pp. 6350-6354, 2014.

[5] Pritika Patel and Pritika Patel, "Review of Different Inpainting Algorithms", International Journal of Computer Applications (IJCA) Vol. 59- No.18, December 2012.

[6] Rajul Suthar and Mr. Krunal R. Patel, "A Survey on Various Image Inpainting Techniques to Restore Image”, International Journal of Engineering Research and Applications, Vol. 4, No. 2, Version 1, pp. 85-88, February 2014.

[7] Nirali Pandya and Bhailal Limbasiya, "A Survey on Image Inpainting Techniques", International Journal of Current Engineering and Technology, 2013.

[8] A Vijayalakshmi and Pethuru Raj, "Modified Exemplar based Image Inpainting Algorithm", International Journal of Computer Applications (IJCA), Vol. 135, No. 6, February 2016.

[9] M. Bertalmio, G. Saprio, V. Caselles, and C. Ballester, "Image Inpainting”, Proceedings of the 27th annual conference on Computer graphics and interactive technique, pp. 417-424, 2000.

[10] R. Mart'inez-Noriega, A. Roumy and G. Blanchard, "ExemplarBased Image Inpainting: Fast Priority and Coherent Nearest Neighbor Search”, 2012 IEEE International Workshop on Machine Learning For Signal Processing, Santander, Spain, Sept. 23-26, 2012

[11] Dong, Bin, Hui Ji, Jia Li, Zuowei Shen, and Yuhong Xu. "Wavelet frame based blind image inpainting", Applied and Computational Harmonic Analysis, Vol. 32, No. 2, pp. 268-279, 2012.

[12] Xie, Junyuan, Linli Xu, and Enhong Chen, "Image denoising and inpainting with deep neural networks", In Advances in neural information processing systems, pp. 341-349. 2012.

[13] Dong, Weisheng, Guangming Shi, and Xin Li. "Nonlocal image restoration with bilateral variance estimation: a low-rank approach", IEEE transactions on image processing, Vol. 22, No. 2, pp. 700-711, 2013.

[14] Hareesh, Anamandra Sai, and Venkatachalam Chandrasekaran. "Exemplar-based color image inpainting: a fractional gradient function approach", Pattern Analysis and Applications, Vol. 17, No. 2, pp. 389-399, 2014.

[15] Qing Zhang and Jiajun Lin, "Exemplar-Based Image Inpainting Using Color Distribution Analysis”, Journal Of Information Science and Engineering, Vol. 28, pp. 641-654, 2012.

[16] Liu, Yunqiang, and Vicent Caselles, "Exemplar-based image inpainting using multiscale graph cuts", IEEE transactions on image processing, Vol. 22, No. 5, pp. 1699-1711, 2013. 